\title{
Влияние температуры фотонного отжига на структурные и оптические свойства пленок ZnO, синтезированных методом дуального магнетронного распыления
}

\author{
(C) С.В. Зайцев, В.С. Ващилин, В.В. Колесник, М.В. Лимаренко, \\ Д.С. Прохоренков, Е.И. Евтушенко
}

Белгородский государственный технологический университет им. В.Г. Шухова, 308012 Белгород, Россия

E-mail: sergey-za@mail.ru

(Получена 13 июня 2018 г. Принята к печати 6 июля 2018 г.)

Пленки $\mathrm{ZnO}$ толщиной 1.4 мкм осаждали на стеклянные подложки методом дуального магнетронного распыления мишеней $\mathrm{Zn}$ в газовой среде аргона и кислорода. Проведены исследования зависимости структурных и оптических характеристик пленок $\mathrm{ZnO}$ от температуры фотонного отжига после осаждения. Установлено что, повышение температуры отжига приводит к повышению степени кристалличности пленок. Электронная микроскопия показала, что осажденное покрытие $\mathrm{ZnO}$ имеет столбчатую структуру, причем отжиг увеличивает плотность микроструктуры и размер кристаллита. Обнаружено, что при температуре отжига $450-650^{\circ} \mathrm{C}$ коэффициент оптического пропускания увеличился до значения $>90 \%$ в спектральной области 400-1100 нм. Экспериментальные результаты показывают, что температура фотонного отжига в вакууме оказывает наибольшее влияние на конечные свойства покрытий $\mathrm{ZnO}$.

DOI: 10.21883/FTP.2019.02.47111.8933

\section{1. Введение}

Интерес к оксиду цинка $(\mathrm{ZnO})$ обусловлен особой комбинацией оптических, электрических и акустических свойств. Кроме того, он имеет большую энергию связи экситона, $\sim 60$ мэВ, и большую ширину запрещенной зоны, 3.36 эВ при комнатной температуре [1]. Благодаря этим свойствам пленки $\mathrm{ZnO}$ применяются в солнечных элементах, детекторах ультрафиолетового и инфракрасного излучения, газовых сенсорах и пьезоэлектронных устройствах в качестве преобразователя поверхностно-акустических волн [2-4].

Пленки $\mathrm{ZnO}$ получают различными методами, такими как химическое осаждение из паровой фазы, импульсное лазерное осаждение, магнетронное распыление, осаждение атомных слоев и молекулярно-лучевая эпитаксия [5-9]. Магнетронное распыление имеет много привлекательных преимуществ, которые позволяют получить покрытия с хорошо воспроизводимыми свойствами, высокую адгезию сформированных пленок с основой, a также осуществлять осаждение при относительно низких температурах, что уменьшает риск образования трещин из-за различия коэффициентов теплового расширения подложки и покрытия [10-14]. Известно, что свойства осажденных пленок $\mathrm{ZnO}$ зависят не только от параметров осаждения, таких как давление, доля реакционного газа и температура подложки $[15,16]$, но и от процессов после осаждения, таких как отжиг [17]. В процессе термического воздействия дислокации и структурные дефекты перемещаются в материале, в результате структура и стехиометрическое соотношение покрытия материала будут меняться. Такие явления могут оказывать существенное влияние на свойства $\mathrm{ZnO}$, что весьма актуально.
В данной работе представлены результаты исследований влияния температуры фотонного отжига в вакууме на микроструктуру и оптические свойства пленок $\mathrm{ZnO}$, синтезированных методом дуального магнетронного распыления.

\section{2. Методика получения и исследования пленок}

\section{1. Осаждение пленок ZnO}

Пленки $\mathrm{ZnO}$ осаждали на подложки из предметного стекла с помощью вакуумной установки QUADRA 500TM методом дуального магнетронного распыления. Перед помещением в камеру установки подложки подвергались ультразвуковой очистке в ацетоне, этаноле, а затем промывались дистиллированной водой и сушились в потоке сухого азота. Вакуумную камеру откачивали до давления $<5 \cdot 10^{-3}$ Па, что достигалось использованием турбомолекулярного насоса. Скорость откачки составляла 1028 л/мин. Перед нанесением покрытия проводилась ионная очистка поверхности образцов в среде аргона (Ar, о.ч. 99.999\%) при напряжении 2000 В, давлении $6.5 \cdot 10^{-2}$ Па, время очистки составляло 10 мин. Покрытия наносились из двух вертикально установленных мишеней (катодов) путем распыления цинка $(\mathrm{Zn}$, чистота 99.999\%) в газовой среде $\mathrm{Ar}$ и кислорода $\left(\mathrm{O}_{2}\right.$, о.ч. $99.999 \%$ ) при давлении 0.22 Па, токе $2 \mathrm{~A}$, время осаждения составляло 60 мин. Скорость вращения карусели с образцами составляла 18 об/мин. Объемная доля реактивного газа $\mathrm{O}_{2}$ составляло $18 \%$. Расстояние от подложек до магнетрона и ионного источника было 
одинаковым, 100 мм. Во время нанесения покрытия подложка дополнительно не подогревалась.

\section{2. Фотонный отжиг после распыления}

Фотонный отжиг пленок $\mathrm{ZnO}$ проводили в технологической установке STE RTP 150 в интервале температур $250-650^{\circ} \mathrm{C}$ с шагом $100^{\circ} \mathrm{C}$. Образцы устанавливали на тепловыравнивающие диски из графита, обеспечивающие однородность нагрева. Камеру установки откачивали до давления ниже 800 Па, затем осушали потоком сухого азота в течение 10 мин с последующим созданием вакуума на уровне остаточного давления 260 Па. Далее проводили градиентный фотонный отжиг пленок в течение 3 мин. Контроль температуры пленок во время процесса отжига осуществлялся с помощью пирометра. После отжига образцы остывали в камере до комнатной температуры.

\section{3. Характеристики пленок}

Кристаллическую структуру пленок $\mathrm{ZnO}$ исследовали с помощью рентгеновской дифракции (ARL X'TRA, ThermoTechno) с источником $\mathrm{Cu} K_{\alpha}$ в диапазоне углов $2 \theta=30-70^{\circ}$ в режиме асимметричной компланарной съемки со скользящим углом падения $\alpha=3^{\circ}$ ( $\theta$-scan $)$ для исключения пиков от подложки. Идентификация фаз и индексирование пиков осуществлялись по базе JCPDF.

Морфологические исследования поверхностей и сколов покрытий проводились с помощью сканирующего электронного микроскопа (TESCAN MIRA 3 LMU). Микрорельеф поверхности пленок $\mathrm{ZnO}$ исследовали с помощью сканирующего зондового микроскопа „NanoEducator" фирмы NT MDT. Оптические свойства пленок в видимом и инфракрасном (ИК) диапазонах регистрировали с помощью спектрофотометра ПЭ-6100УФ и модуляционного спектрального эллипсометра Uvisel-2 (Horiba Jobin Yvon).

\section{3. Экспериментальные результаты и обсуждение}

\section{1. Структурные характеристики}

На рис. 1 представлены дифрактограммы покрытий $\mathrm{ZnO}$ как в состоянии после осаждения, так и термически обработанных при различных температурах. По результатам рентгеновской дифракции синтезированное покрытие относится к поликристаллическому $\mathrm{ZnO}$ со структурой вюрцита.

Анализ картины дифракции (рис. 1) показывает, что все пленки $\mathrm{ZnO}$ имеют предпочтительную кристаллографическую ориентацию вдоль оси с, т.е. плоскость (002) перпендикулярна подложке. Фотонный отжиг оказывает влияние на интенсивность дифракционного пика (002), что указывает на рост зерна. Положение дифракционного пика (002) пленок $\mathrm{ZnO}$ сдвигается в меньшие углы $2 \theta$

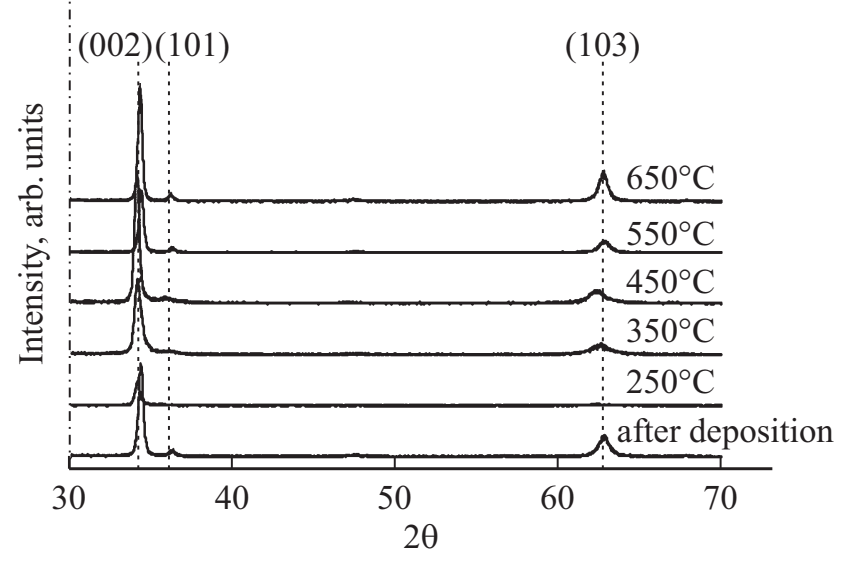

Рис. 1. Дифрактограммы пленок $\mathrm{ZnO}$, после осаждения и после отжига при различных температурах (температуры указаны).

от 34.32 до $34.06^{\circ}$ после фотонного отжига в интервале температур $250-450^{\circ} \mathrm{C}$, а для пленок, отожженных при более высоких температурах, 550 и $650^{\circ} \mathrm{C}$, угол возрастает с 34.32 до $34.38^{\circ}$. Как утверждают авторы работы [18], подобное изменение связано с внутренними напряжениями, возникающими из-за дефектов (промежуточных соединений цинка или вакансий кислорода) в решетке $\mathrm{ZnO}$.

На рис. 2 приведена морфологическая картина поверхности и фрактограммы покрытий $\mathrm{ZnO}$ на подложке из стекла до и после фотонного отжига в вакууме в интервале температур $250-650^{\circ} \mathrm{C}$ в течение 3 мин. Из анализа фрактограммы излома (рис. 2, $a$, вставка) видно, что слой $\mathrm{ZnO}$ на стекле имеет столбчатую структуру из зерен, ориентированных перпендикулярно подложке. После фотонного отжига в вакууме происходит рекристаллизация, но сохраняется столбчатая структура (рис. 2, $b, c, d, e, f$, вставка). Морфология поверхности (рис. 2) показывает, что синтезированные и термически обработанные пленки $\mathrm{ZnO}$ имеют нанокристаллическую структуру без видимых пор и сформированы в виде пирамидальных кристаллитов с одинаковым направлением роста. Следует отметить, что размер зерна и плотность упаковки увеличиваются при повышении температуры фотонного отжига. Средние размеры зерна и толщины

Таблица 1. Характеристика покрытий

\begin{tabular}{c|c|c|c}
\hline $\begin{array}{c}\text { Температура } \\
\text { отжига, }{ }^{\circ} \mathrm{C}\end{array}$ & $\begin{array}{c}\text { Толщина } \\
\text { покрытия, нм }\end{array}$ & $\begin{array}{c}\text { Средний } \\
\text { размер } \\
\text { зерна, нм }\end{array}$ & $\begin{array}{c}\text { Средняя } \\
\text { шероховатость } \\
\text { поверхности, нм }\end{array}$ \\
\hline После осаждения & 1463 & 88 & 6.44 \\
250 & 1450 & 106 & 4.53 \\
350 & 1457 & 109 & 7.34 \\
450 & 1463 & 122 & 8.65 \\
550 & 1450 & 116 & 8.08 \\
650 & 1450 & 102 & 6.85
\end{tabular}


$a$
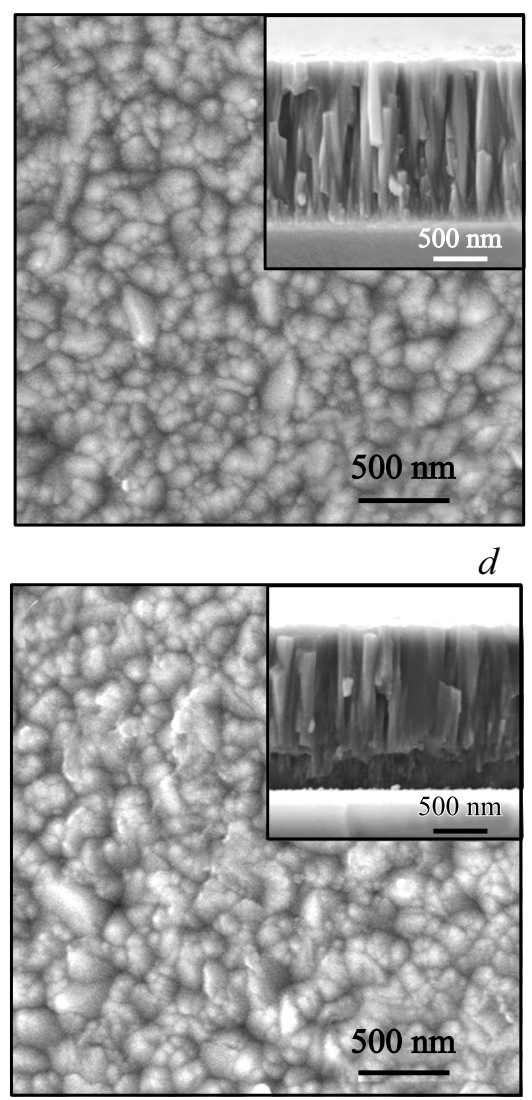

$b$
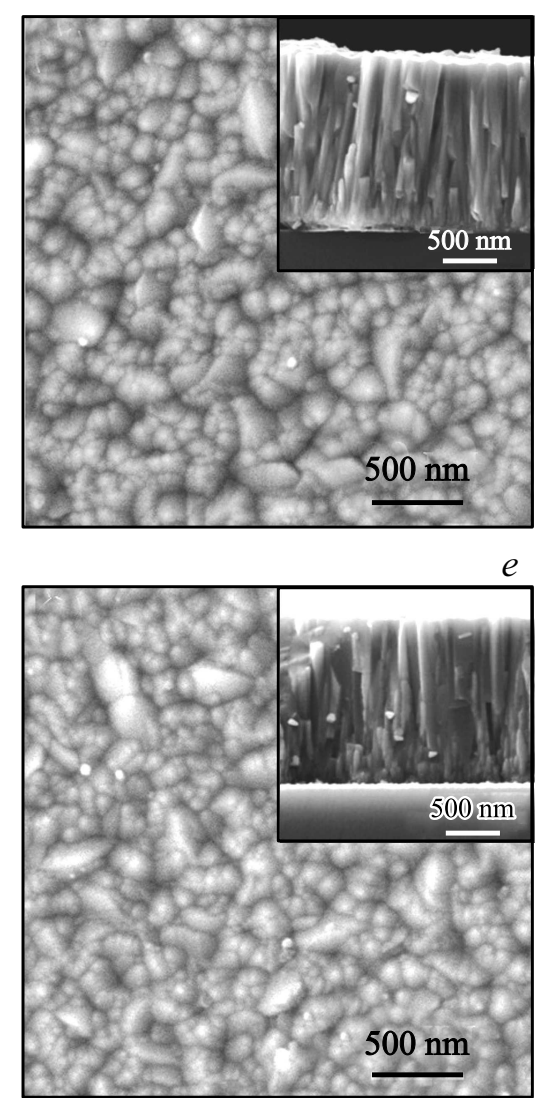

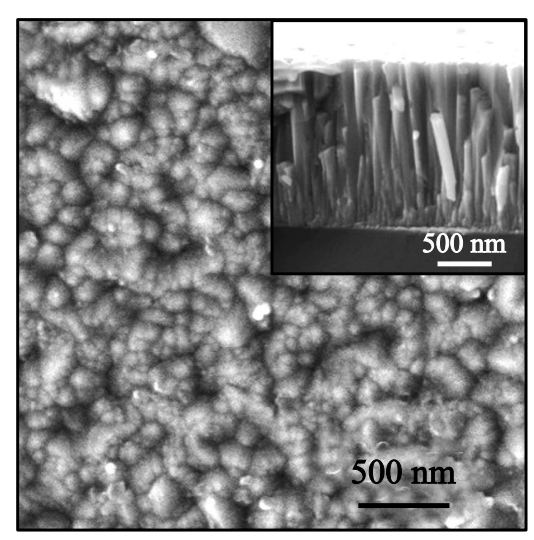

$f$

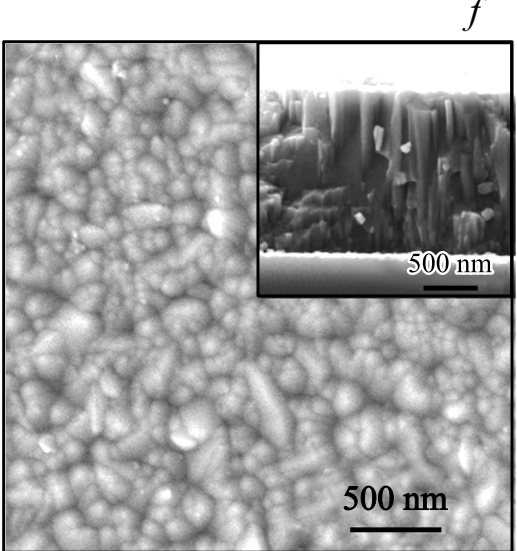

Рис. 2. Морфология поверхности и поперечное сечение (на вставках) пленок ZnO после осаждения (a) и термически обработанных при температурах $250(b), 350(c), 450(d), 550(e), 650^{\circ} \mathrm{C}(d)$.

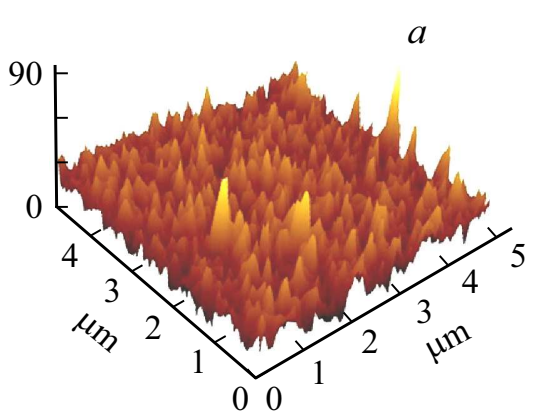

$d$

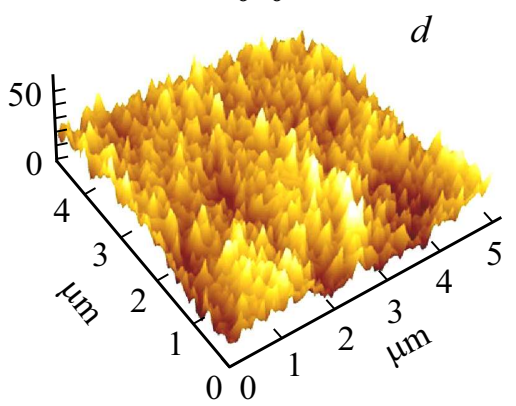

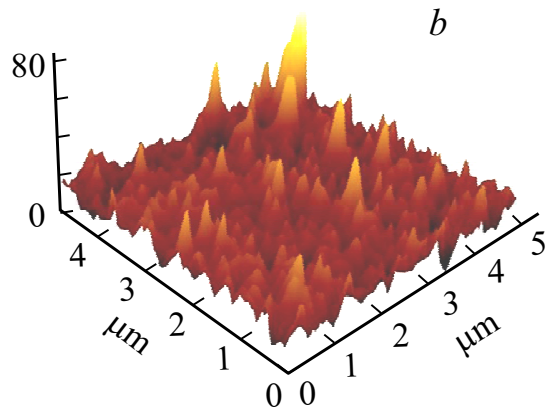

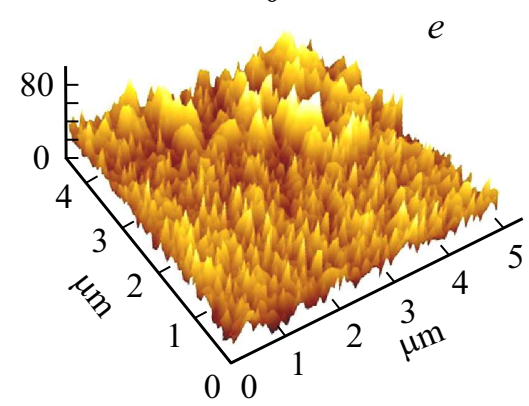

$c$

\section{.}

(1)

\section{at"}


покрытий, определенные по данным микроскопии, представлены в табл. 1.

Увеличение среднего размера зерен с повышением температуры отжига может быть обусловлено срастанием маленьких кристаллитов $\mathrm{ZnO}$ по границам зерна в более крупные кристаллиты. Так, при температурном отжиге дефекты пленки $\mathrm{ZnO}$ на границах зерен стимулируют процесс слияния большего количества зерен, т. е. коалесценцию, что приводит к росту размера зерна [19].

На рис. 3 показан микрорельеф поверхности пленок $\mathrm{ZnO}$ в зависимости от температуры отжига. Топографические исследования поверхности пленок $\mathrm{ZnO}$ показывают, что значения шероховатости этих пленок находились между 4.53 и 8.65 нм, показывая хорошую однородность пленок. Шероховатость поверхности достигает максимального значения 8.65 нм для образца, отожженного при $450^{\circ} \mathrm{C}$. Однако следует отметить, что фотонный отжиг в вакууме слабо влияет на шероховатость поверхности.

\section{2. Оптические характеристики}

Одним из важных параметров применения пленок $\mathrm{ZnO}$ является большое значение оптической проницаемости. На рис. 4 показаны спектры пропускания в диапазоне длин волн $300-1100$ нм пленок $\mathrm{ZnO}$ после синтеза и при отжиге $250-650^{\circ} \mathrm{C}$ в вакууме. Все измерения осуществлялись при комнатной температуре.

Из рис. 4 видно, что пленки $\mathrm{ZnO}$ не пропускают излучение в ультрафиолетовой области. Пропускание начинается в видимой области спектра с 380 нм и достигает максимальных значений для осажденной и отожженных при 250 и $350^{\circ} \mathrm{C}$ пленок $<40 \%$, а для отожженных при 450,550 и $650^{\circ} \mathrm{C}-$ более $90 \%$ в видимом и ближнем ИК диапазонах. Коэффициент пропускания пленок $\mathrm{ZnO}$ возрастает в тандеме с температурой фотонного отжига из-за увеличения размеров зерна, структурной однородности и кристалличности. О том же наблюдении сообщили предыдущие исследователи [20,21].

Оптическую ширину запрещенной зоны определяли в соответствии с законом Тауца, используя аппроксима-

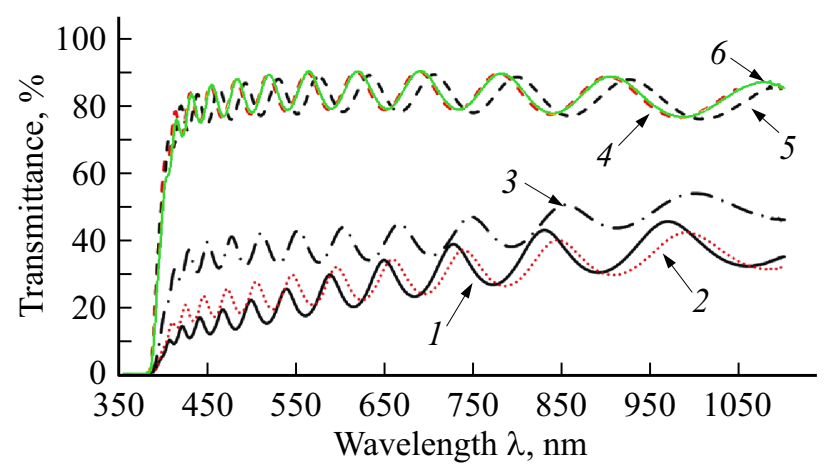

Рис. 4. Спектры оптического пропускания пленок $\mathrm{ZnO}$ после осаждения (1) и после отжига при температурах 250 (2), $350(3), 450(4), 550(5), 650^{\circ} \mathrm{C}(6)$.

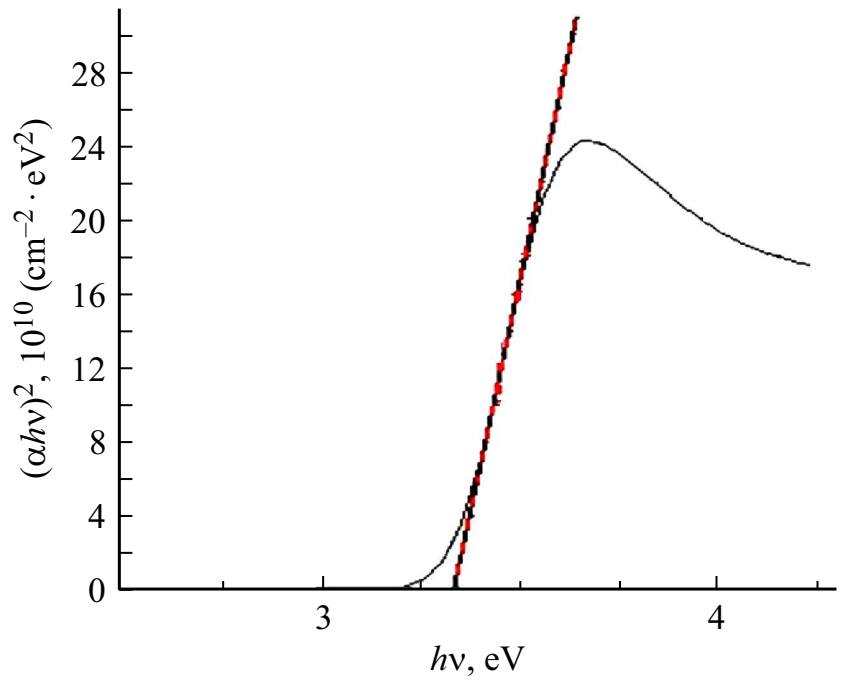

Рис. 5. Спектральная зависимость квадрата коэффициента поглощения от энергии фотонов для пленок $\mathrm{ZnO}$.

цию края поглощения [22]

$$
\alpha h v=A\left(h v-E_{g}\right)^{n},
$$

где $\alpha-$ коэффициент поглощения материала, $A-$ константа для прямого перехода, $h v-$ энергия падающего фотона, $E_{g}$ - ширина запрещенной зоны. Предыдущие работы $[23,24]$ показали, что показатель степени $n=1 / 2$, так как наиболее близко подходит к теоретическому значению ширины запрещенной зоны $E_{g}$.

Из рис. 5 методом экстраполяции линейных участков соответствующих зависимостей $(\alpha h v)^{2}=f(h v)$ до пересечения с осью энергий $h v$ определяют ширину запрещенной зоны пленок $\mathrm{ZnO}$. Значения ширины запрещенной зоны пленок, сформированных на подложке из предметного стекла и с последующим фотонным отжигом в вакууме, представлены в табл. 2 . Видно, что фотонный отжиг в вакууме приводит к изменению ширины запрещенной зоны покрытий $\mathrm{ZnO}$. Это изменение оптической ширины запрещенной зоны можно объяснить уменьшением структурных дефектов в пленках и увеличением размера зерна при отжиге [25].

Таблица 2. Ширина запрещенной зоны в зависимости от температуры отжига $\mathrm{ZnO}$

\begin{tabular}{c|c}
\hline $\begin{array}{c}\text { Температура } \\
\text { отжига, }{ }^{\circ} \mathrm{C}\end{array}$ & $\begin{array}{c}\text { Ширина запрещенной } \\
\text { зоны } E_{g}, \text { эВ }\end{array}$ \\
\hline После осаждения & 3.31 \\
250 & 3.49 \\
350 & 3.26 \\
450 & 3.31 \\
550 & 3.31 \\
650 & 3.31
\end{tabular}




\section{4. Заключение}

Исследовано влияние температуры фотонного отжига в вакууме на оптические свойства и структуру пленок $\mathrm{ZnO}$, осажденных методом дуального магнетронного распыления мишени $\mathrm{Zn}$ в газовой среде $\mathrm{Ar}-\mathrm{O}_{2}$. Спектры рентгеновской дифракции показали, что пленки имеют гексагональную структуру вюрцита с предпочтительной кристаллографической ориентацией (002). Установлено, что качество кристаллитов зависит от температуры фотонного отжига и проявляется в изменении интенсивности отражений на дифрактограммах.

Из анализа электронной микроскопии установлено, что осажденное покрытие $\mathrm{ZnO}$ состоит из столбчатой структуры, причем фотонный отжиг увеличивает плотность микроструктуры и размер кристаллита. Исследование микрорельефа поверхности пленок $\mathrm{ZnO}$ показало, что средние значения шероховатости незначительно зависят от температуры фотонного отжига.

Показано что, фотонный отжиг в вакууме влияет на оптические свойства пленок $\mathrm{ZnO}$. Так, отжиг при температуре $450-650^{\circ} \mathrm{C}$ позволяет получить покрытия с коэффициентом оптического пропускания $>90 \%$ в спектральной области 400-1100 нм, пригодные для применения в приборах оптоэлектроники.

Анализ полученных экспериментальных данных показал, что фотонный отжиг пленок $\mathrm{ZnO}$ в вакууме является эффективным методом управления их оптическими и структурными свойствами.

Работа выполнена в рамках государственного задания Министерства образования и науки РФ № $11.9329 .2017 / 8.9$, с использованием оборудования ЦВТ БГТУ им. В.Г. Шухова.

\section{Список литературы}

[1] V. Senay, S. Pat, S. Korkmaz, T. Aydogmus, S. Elmas, S. Özen, N. Ekem, M.Z. Balbag. Appl. Surf. Sci., 318, 2 (2014).

[2] V.S. Burakov, N.V. Tarasenko, E.A. Nevar, M.I. Nedel'ko. Technical Physics, 56 (2), 245 (2011).

[3] D.T. Phan, G.S. Chung. Appl. Surf. Sci., 257 (9), 4339 (2011).

[4] Y. Natsume, H. Sakata. J. Mater. Sci.: Materials in Electronics, $12(2), 87$ (2001).

[5] Z. Li, Z. Hu, L. Jiang, H. Huang, F. Liu, X. Zhang, Y. Wang, P. Yin, L. Guo. Mater. Lett., 79, 209 (2012).

[6] M. Suchea, S. Christoulakis, C. Tibeica, M. Katharakis, N. Kornilios, T. Efthimiopoulos, E. Koudoumas. Appl. Surf. Sci., 254 (17), 5475 (2008).

[7] В.Б. Залесский, Т.Р. Леонова, О.В. Гончарова, И.А. Викторов, В.Ф. Гременок, Е.П. Зарецкая. Физика и химия твердого тела, 6 (1), 44 (2005).

[8] K.W. Kim, H.S. Son, N.J. Choi, J. Kim, S.N. Lee. Thin Sol. Films, 546, 114 (2013).

[9] B. Zhang, B. Yao, S. Wang, Y. Li, C. Shan, J. Zhang, B. Li, Z. Zhang, D. Shen. J. Alloys Comp., 503 (1), 155 (2010).
[10] А.И. Кузьмичев. Магнетронные распылительные системы (М., Изд-во Аверс, 2008).

[11] D. Manova, J.W. Gerlach, S. Mändl. Materials, 3 (8), 4109 (2010).

[12] J.R.R. Bortoleto, M. Chaves, A.M. Rosa, E.P. da Silva, S.F. Durrant, L.D. Trino, P.N. Lisboa-Filho. Appl. Surf. Sci., 334, 210 (2015).

[13] В.М. Нарцев, М.С. Агеева, Д.С. Прохоренков, С.В. Зайцев, С.В. Карацупа, В.С. Ващилин. Вестн. БГТУ им. В.Г. Шухова, 6, 168 (2013).

[14] С.В. Зайцев, В.М. Нарцев, В.С. Ващилин, Д.С. Прохоренков, Е.И. Евтушенко. Российские нанотехнологии, $11(5-6), 18$ (2016).

[15] C.W. Hsu, T.C. Cheng, C.H. Yang, Y.L. Shen, J.S. Wu, S.Y. Wu. J. Alloys Comp., 509 (5), 1774 (2011).

[16] Y.Y. Kim, S.W. Kang, B.H. Kong, H.K. Cho. Physica B: Condens. Matter, 401, 408 (2007).

[17] A. Purohit, S. Chander, A. Sharma, S.P. Nehra, M.S. Dhaka. Optical Mater., 49, 51 (2015).

[18] G.A. Kumar, M.R. Reddy, K.N. Reddy. J. Physics: Conf. Ser., 365 (1), 012031 (2012). http://iopscience.iop.org/ article/10.1088/1742-6596/365/1/012031/meta

[19] Z.B. Fang, Z.J. Yan, Y.S. Tan, X.Q. Liu, Y.Y. Wang. Appl. Surf. Sci., 241 (3-4), 303 (2005).

[20] S.U. Lee, B. Hong, J.H. Boo. Funct. Mater. Lett., 3 (02), 119 (2010).

[21] A.L. Mercado, C.E. Allmond, J.G. Hoekstra, J.M. Fitz-Gerald. Appl. Phys. A, 81 (3), 591 (2005).

[22] G.P. Daniel, V.B. Justinvictor, P.B. Nair, K. Joy, P. Koshy, P.V. Thomas. Physica B: Condens. Matter, 405(7), 1782 (2010).

[23] O. Lupan, T. Pauporté, L. Chow, B. Viana, F. Pellé, L.K. Ono, B.R. Cuenya, H. Heinrich. Appl. Surf. Sci., 256 (6), 1895 (2010).

[24] E.N. Cho, S. Park, I. Yun. Current Appl. Phys., 12 (6), 1606 (2012).

[25] M.F. Malek, M.H. Mamat, M.Z. Musa, Z. Khusaimi, M.Z. Sahdan, A.B. Suriani, A. Ishak, I. Saurdi, S.A. Rahman, M. Rusop. J. Alloys Comp., 610, 575 (2014).

Редактор Л.В. Шаронова 


\section{The influence of photon annealing temperature on structural and optical properties of $\mathrm{ZnO}$ films synthesized by the dual magnetron sputtering method}

S.V. Zaitsev, V.S. Vaschilin, V.V. Kolesnik, M.V. Limarenko, D.S. Prokhorenkov, E.I. Evtushenko

Belgorod State Technological University named after V.G. Shukhov, 308012 Belgorod, Russia

Abstract $\mathrm{ZnO}$ films $1.4 \mu \mathrm{m}$ thick were deposited on glass substrates by the method of dual magnetron sputtering of $\mathrm{Zn}$ targets in argon and oxygen gas atmosphere. The dependence of the structural and optical characteristics of $\mathrm{ZnO}$ films on the photon annealing temperature after deposition is studied. It is established that an increase in the annealing temperature leads to improvement in the crystallinity of the films. Electron microscopy showed that the precipitated $\mathrm{ZnO}$ coating had a columnar structure, with annealing its density and crystallite sizes increased. It was found that at the annealing temperature of $450-650^{\circ} \mathrm{C}$ the optical transmission coefficient increased and was more than $90 \%$ in the spectral region of $400-1100 \mathrm{~nm}$. The experimental results showed that the temperature of photon annealing in vacuum had the greatest effect on the final properties of $\mathrm{ZnO}$ coatings. 